\title{
Acquired Resistance to Targeted MAPK Inhibition in Melanoma
}

\author{
Kavitha Gowrishankar, Matteo S. Carlino and \\ Helen Rizos \\ Additional information is available at the end of the chapter \\ http://dx.doi.org/10.5772/53629
}

\section{Introduction}

The worldwide incidence of cutaneous melanoma has steadily increased in fair-skinned individuals over recent decades with estimates suggesting a doubling of melanoma incidence every 10-20 years [1]. Melanoma remains the major cause of skin cancer related deaths [2], with survival rates averaging less than six months for patients with metastases in visceral organs [3]. Conventional systemic therapies, including single agent dacarbazine and temozolamide, produce response rates of less than $10 \%$, and are not proven to improve survival (reviewed in [4]). Recently, however, the treatment of melanoma has been revolutionized by therapies targeting the RAF-MEK-ERK mitogen activated protein kinase (MAPK) pathway. This pathway is constitutively activated in the majority of melanomas via oncogenic mutations in the BRAF kinase or its upstream regulator, N-RAS [5, 6].

Most BRAF mutations produce a single amino acid substitution of valine by glutamic acid at amino acid 600 (V600E), and this leads to a 500-fold increase in kinase activity [5, 7]. Targeting this mutant BRAF with the highly specific inhibitors, vemurafenib (PLX4032) and dabrafenib (GSK2118436) has produced response rates above 50\% and improved progression-free survival in patients with BRAF-mutant metastatic melanoma [8-12]. Both BRAF inhibitors are active against melanoma brain metastases $[13,14]$ and vemurafenib treatment prolongs overall survival compared with dacarbazine [11]. Despite the marked initial responses to BRAF inhibitors, tumor re-growth occurs in most patients with a median progression-free survival of 5 to 6 months $[8,11,15]$.

The U.S Food and Drug Administration (FDA) approved the use of vemurafenib for the treatment of BRAF-mutant melanoma in 2011, and submissions for the use of dabrafenib in 
the treatment of BRAF-mutant melanoma were made in late 2012. We are now beginning to understand the complex pathways regulating the response and side-effect profiles of these targeted inhibitors. The challenge is to define the molecular drivers and pathways of resistance and response and to translate these molecular findings into rational strategies for clinical testing and improved therapies. In the following chapter we describe the molecular mechanisms that contribute to BRAF inhibitor resistance in vitro and in vivo. We also highlight the current strategies employed to dissect resistance drivers and explore the future of targeted therapies in the long-term treatment of melanoma.

\section{The BRAF kinase and the MAPK pathway}

Aberrant activation of the MAPK pathway is present in over $80 \%$ of primary cutaneous melanomas [16]. MAPK signalling is driven by mutated N-RAS and activating mutations in the downstream RAS effector, BRAF, in $20 \%$ and $60 \%$ of melanomas, respectively (Figure 1) [16]. Of cutaneous melanomas with no mutations in BRAF or N-RAS, many activate MAPK signalling via oncogenic mutations in the receptor tyrosine kinase, c-Kit [17], activating mutations in the Rac1 GTPase or inactivating mutations in the N-RAS inhibitor NF1 [18].

Among the BRAF mutations identified in melanoma, over $80 \%$ involve a single nucleotide mutation resulting in the substitution of valine for glutamic acid at amino acid 600. This mutation is also present in up to $80 \%$ of benign, growth-arrested nevi [19], implicating BRAF as an initiating event that co-operates with additional genetic lesions to promote melanoma. Over 60 other mutations in BRAF have been described in melanoma; most affect codon 600 (V600E, V600K, V600R and V600D), lie within the kinase domain and show elevated kinase activity. In particular, alterations affecting codon 600 show 150- (BRAFV600K) to 700$\left(\mathrm{BRAF}^{\mathrm{V} 600 \mathrm{D}}\right.$ ) fold more kinase activity than the wild type BRAF protein [7].

A wealth of preclinical data has demonstrated the critical role of $\mathrm{BRAF}^{\mathrm{V} 600 \mathrm{E}}$ as an oncogene in melanoma. The specific silencing of BRAF with short interfering (si)RNA resulted in decreased ERK signalling, diminished proliferation and regression of BRAF mutant melanomas [20-23]. More importantly, class I RAF inhibitors, which target the activated form of RAF kinases, show remarkable antitumor activity; both vemurafenib and dabrafenib have shown response rates of $50 \%$ in patients with BRAF-mutant melanoma [8-12]. In addition, the selective inhibition of the BRAF target proteins, MEK1/2, with trametinib (GSK11202212) improved rates of progression-free and overall survival amongst patients with BRAF mutant melanoma when compared to dacarbazine [9, 15, 24, 25].

\section{Mechanisms of acquired BRAF inhibitor resistance in melanoma}

Despite the marked initial responses to single-agent BRAF inhibitors, tumor re-growth occurs in most patients and $5-20 \%$ of individuals fail to respond early during treatment $[8,10$, $11,26]$. The acquisition of resistance to targeted therapy is common and resistance has been 
observed with trastuzumab in HER2-amplified breast cancer, imatinib in gastrointestinal stromal tumors (GISTs) and chronic myelogenous leukemia (CML), epidermal growth factor receptor (EGFR) inhibitors in lung cancer and hedgehog inhibitors in medulloblastoma [27]. Resistance mechanisms to these drugs are complex but include the acquisition of secondary mutations in the target oncogene that prevent drug binding, up-regulation of signalling pathways downstream of the target and the induction of alternate, secondary survival pathways. Defining the mechanisms of melanoma resistance to targeted inhibitors is a high priority, as it can guide the selection of appropriate drug combinations and advance the development of new and improved drugs. This is best demonstrated for imatinib-resistant leukemias. The identification of secondary Bcr-Abl mutations in these resistant cancers promoted the development of the potent, next-generation receptor tyrosine kinase inhibitors dasatanib and nilotinib [28].

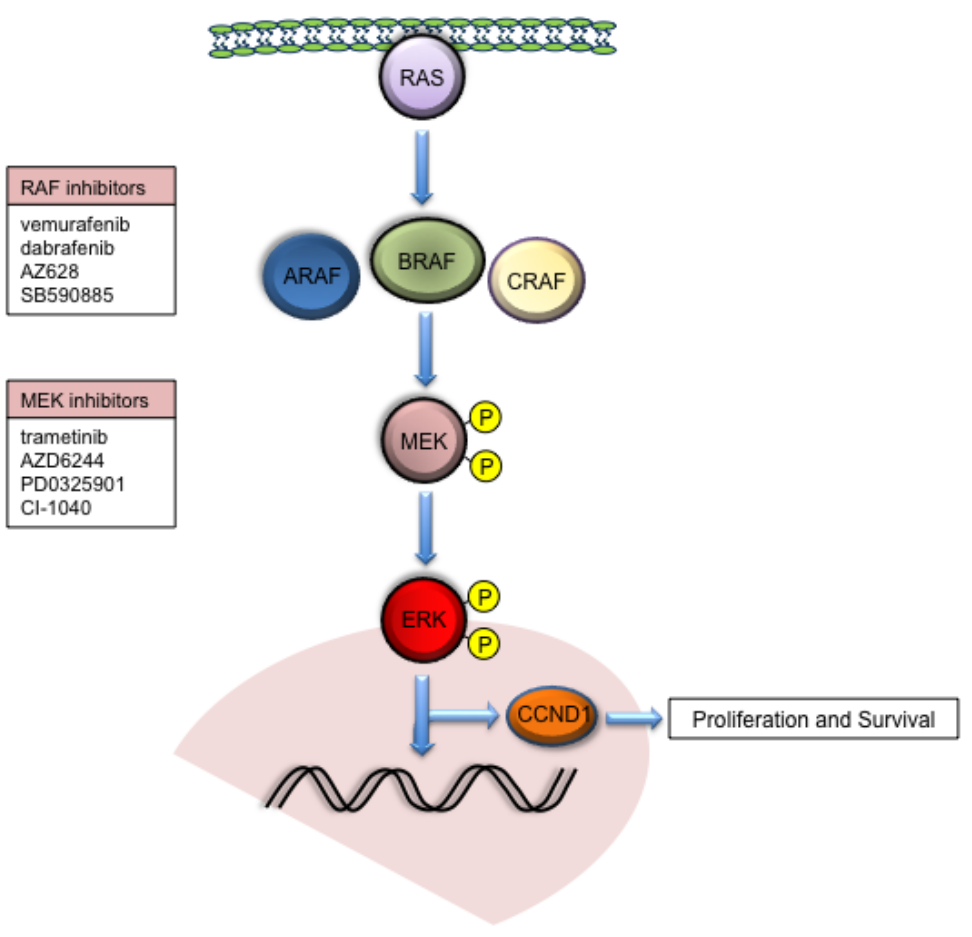

Figure 1. MAPK signalling cascade. Activation of the RAS GTPase promotes the kinase activity of the RAF serine/ threonine protein kinases, ARAF, BRAF and CRAF. Activated RAF kinases promote the sequential phosphorylation and activation of the MEK1/2 and ERK1/2 kinases. The ERK proteins translocate into the nucleus and stimulate the translation of proteins and the activities of many transcription factors. This leads to a series of gene expression changes, including elevated CCND1 that promotes cell proliferation and survival. Specific inhibitors to RAF and MEK kinases are indicated. ARAF, v-raf murine sarcoma 3611 viral oncogene homolog; BRAF, v-raf murine sarcoma viral oncogene B1; CCND1, cyclin D1; CRAF, v-raf-1 murine leukemia viral oncogene homolog 1; ERK, extracellular signal-regulated kinase; MEK mitogen-activated protein kinase kinase. 


\section{Alterations affecting BRAF}

Drug resistance is often associated with the acquisition of so-called gatekeeper mutations in the target oncogene that prevent drug binding. In a series of detailed reports, deep sequencing of melanoma biopsies derived from patients progressing on vemurafenib treatment did not find any secondary BRAF mutations. Moreover, immunoprecipitated BRAF from vemurafenib-resistant melanomas retained drug sensitivity in an in vitro kinase assay, confirming drug-target binding was maintained [29].

\subsection{BRAF copy number gain}

The amplification and overexpression of $\mathrm{BRAF}^{\mathrm{V} 600 \mathrm{E}}$ is associated with BRAF inhibitor resistance in melanoma [30] (Figure 2). In a sequencing screen of 20 pairs of patient-matched baseline (before BRAF inhibitor therapy) and progressing (acquired resistance to BRAF inhibition) melanoma tissue, 20\% showed BRAF ${ }^{\mathrm{V} 600 \mathrm{E}}$ copy-number gains, ranging from 2- to 14- fold. These copy-number gains, which are likely underestimates due to non-tumor cell contamination, correlated with increased BRAF protein expression in tumor specimens. Moreover, preclinical melanoma cell models with ectopically expressed $\mathrm{BRAF}^{\mathrm{V} 600 \mathrm{E}}$ confirmed that cells overexpressing mutant BRAF developed resistance to vemurafenib and that this resistance could be overcome by increasing the dose of vemurafenib, applying MEK inhibitors (AZD6244) or concurrently inhibiting both MEK and BRAF (Figure 1) [30].

Unlike melanoma cell models [30], BRAF-mutant colorectal cancer cells with amplification of the BRAF gene (2- to 7- fold) were resistance to the MEK inhibitor AZD6244 [31]. In these colon cancer cells, the increased expression of mutant BRAF resulted in excess activation of MEK and ERK, rendering cells unresponsive to MEK inhibition. In the presence of the BRAF inhibitor, AZ628, however, the abundance of activated MEK was reduced and the allosteric MEK inhibitor AZD6244 prevented ERK phosphorylation [31]. Thus, the concurrent inhibition of MEK and BRAF overcomes resistance mediated by BRAF amplification in both melanoma and colorectal cancers.

Intriguingly, BRAF copy-number gains (3- to 4-fold) were also identified in baseline (drugnaive) melanoma and colorectal tumor samples. In one such colorectal tumor only $28 \%$ of cells showed BRAF amplification and $10 \%$ of these tumor cells had more than 10 copies of BRAF [30, 31]. These data indicate that cell sensitivity to MEK and BRAF inhibition is likely to reflect the level of BRAF amplification and resistance may arise from the expansion of a limited number of cells with pre-existing BRAF gains. This notion is consistent with a recent study showing that K-RAS mutations conferring resistance to EGFR inhibitors were likely to be present in a clonal subpopulation of the colorectal tumor cells prior to the initiation of targeted therapy. These results may explain resistance to RAF inhibitors and other targeted therapies occurs in a highly reproducible fashion within 5 to 6 months [32]. 


\subsection{BRAF splicing variants}

In other melanomas, resistance to vemurafenib was acquired via the expression of splice variant isoforms of BRAFV600E. Three of five vemurafenib-resistant clones of the SKMEL-238 melanoma cells expressed a novel $61 \mathrm{kDa}$ variant of BRAF ${ }^{\mathrm{V} 600 \mathrm{E}}$. This $\mathrm{p} 61 \mathrm{BRAF}{ }^{\mathrm{V} 600 \mathrm{E}}$ splice variant, lacked exons 4-8, a region encoding the RAS binding domain, and was sufficient to render MEK activation resistant to vemurafenib (Figure 3). The variant appears to arise from a splicing defect as no intragenic somatic deletions within the BRAF gene were detected [33].

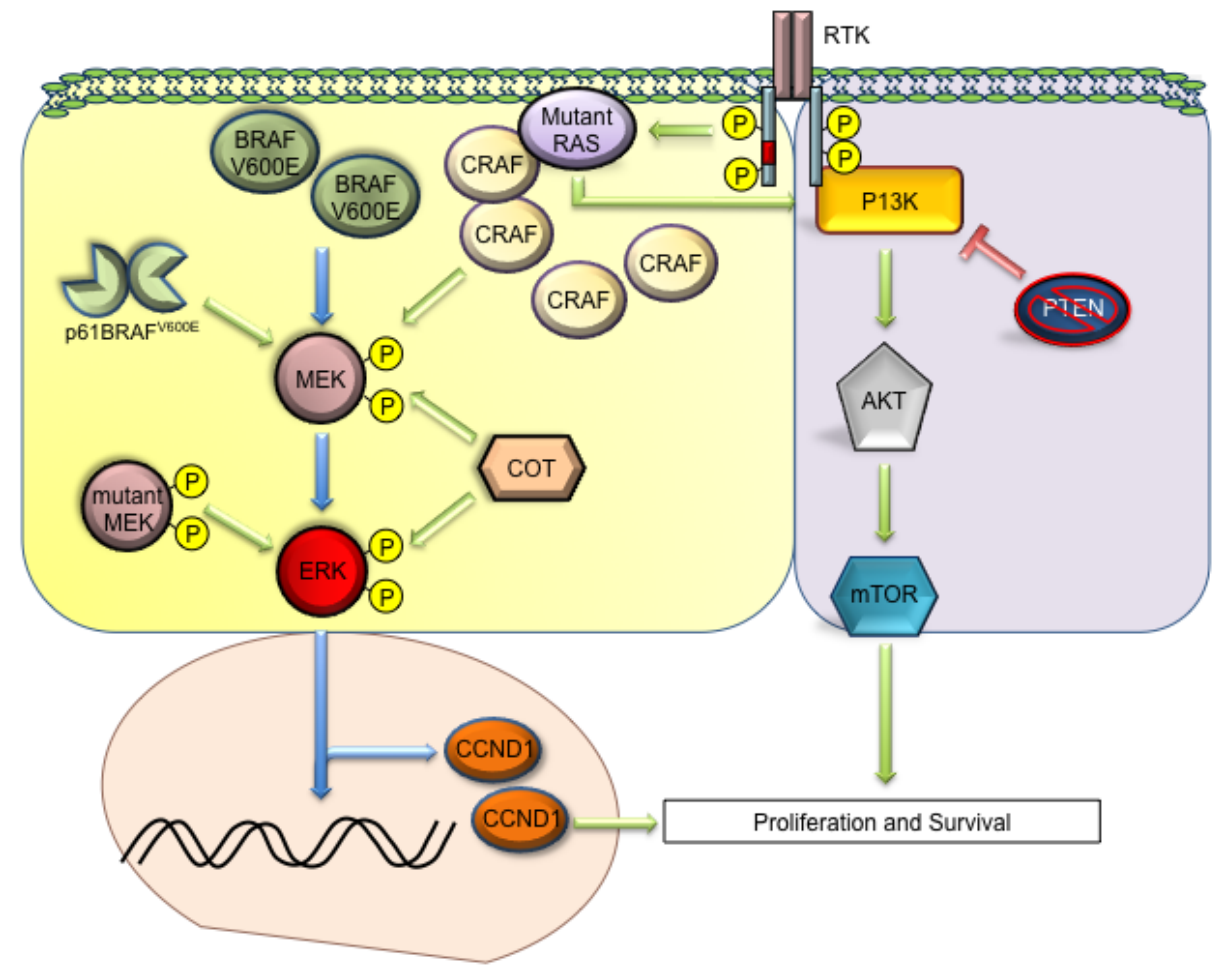

Figure 2. Mechanisms of resistance to BRAF inhibition. MAPK re-activation, in the presence of RAF inhibitors, can occur via $A$. the mutational activation and amplification of RAS, B. the upregulation of RTKs such as PDGFRB and IGF-1R, C. elevated expression of CRAF, COT or CCND1, D. MEK mutations, or E. the expression and dimerization of BRAF $^{\mathrm{V} 600 \mathrm{E}}$ splice variants, such as p61BRAFV600E. Mutant RAS and upregulated RTKs also activate the PI3K/mTOR survival pathway, which is further activated by the loss of PTEN (adapted from [87]). AKT, v-akt murine thymoma viral oncogene; BRAF, v-raf murine sarcoma viral oncogene B1; CCND1, cyclin D1; CRAF, v-raf-1 murine leukemia viral oncogene homolog 1; ERK, extracellular signal-regulated kinase; COT, mitogen-activated protein kinase kinase kinase 8; MEK mitogen-activated protein kinase kinase; mTOR, mechanistic target of rapamycin; PI3K, phosphoinositide-3-kinase PTEN, phosphatase and tensin homolog; RTK, receptor tyrosine kinase. 

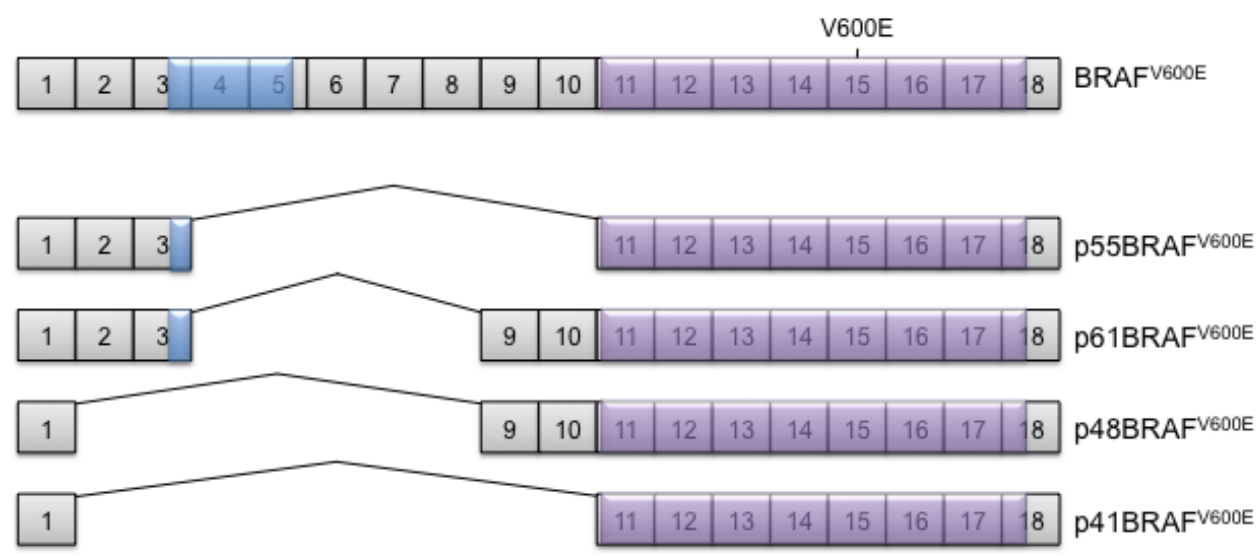

\section{Kinase domain}

RAS binding domain

Figure 3. Aberrant splicing of BRAF ${ }^{\mathrm{V} 600 \mathrm{E}}$ confers RAF inhibitor resistance. Several in frame BRAF ${ }^{\mathrm{V} 600 \mathrm{E}}$ splice variants lacking the RAS binding domain have been detected in vemurafenib-resistant melanoma tumor specimens. The exon structure of full-length and spice variant forms of BRAF are shown. The location of the activating V600E mutation, the RAS binding and kinase domains are highlighted (adapted from [33]).

It is known that the amino terminus of BRAF negatively regulates its kinase activity by masking the carboxy-terminal catalytic domain. Upon binding to activated RAS, the aminoterminal regulatory domain of RAF proteins unfolds to expose the carboxy terminal sites that are required for dimerization and full kinase activity. The in-frame deletion in the p61BRAF ${ }^{\mathrm{V} 600 \mathrm{E}}$ variant leads to the constitutive dimerization of BRAF in the absence of activated RAS [34]. Dimerization of p61BRAF ${ }^{\mathrm{V} 600 \mathrm{E}}$ was shown to be critical for mediating BRAFinhibitor resistance, as the $\mathrm{R} 509 \mathrm{H}$ dimerization-deficient mutant form of p61BRAF ${ }^{\mathrm{V} 600 \mathrm{E}}$ was sensitive to vemurafenib and monomeric p61BRAF ${ }^{\mathrm{V} 600 \mathrm{E}}$ was inhibited by vemurafenib in vitro. Thus, it has been proposed that binding of vemurafenib to one p61BRAF ${ }^{\mathrm{V} 600 \mathrm{E}}$ protomer elicits an allosteric change in the other, drug-free protomer, thereby decreasing its affinity for the drug (Figure 4). Four independent BRAF splicing variants were also detected in tumors derived from six of nineteen patients with acquired resistance to vemurafenib (Figure 3). In each case, the alternative splice variants were in frame, lacked the RAS binding domain and were confined to the mutant allele [33]. This indicates that BRAF missplicing is likely due to a mutation or epigenetic change that specifically impacts the BRAF ${ }^{\mathrm{V} 600 \mathrm{E}}$ allele. Importantly, no splice BRAF variants were detected in 27 melanomas resected from vemurafenib-naive patients [33]. 


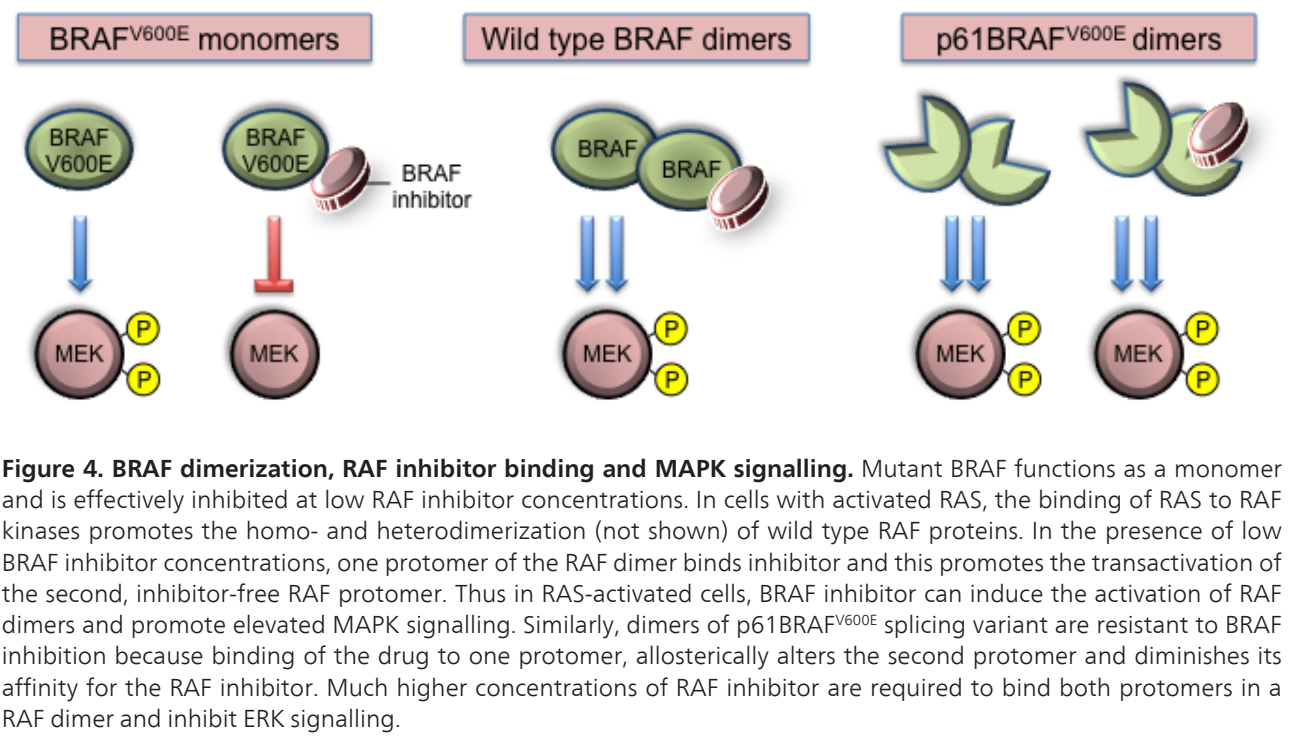

\section{N-RAS mutations}

In the normal physiological setting, activated RAS signalling promotes the dimerization and activation of RAF proteins. In the presence of BRAF inhibitors and RAS signalling, the binding of drug to one molecule in a non-mutated RAF dimer can promote activation of the second RAF molecule (Figure 4). Thus, in the presence of RAS activation, the activity of homoand heterodimeric RAF complexes can be paradoxically activated by RAF inhibitors [35-37].

In melanomas with $\mathrm{BRAF}^{\mathrm{V} 600 \mathrm{E}}$, any alterations promoting RAF dimerization are predicted to confer resistance to RAF inhibitors. As expected, activating N-RAS mutations mediate resistance to vemurafenib [29] and dabrafenib [38]. Oncogenic N-RAS ${ }^{\mathrm{Q} 61 \mathrm{~K}}$ was detected in a single vemurafenib-resistant clone derived from the M249 melanoma cells. This resistant subclone maintained ERK activation in the presence of vemurafenib, presumably via a kinase switch from BRAF to CRAF (Figure 1) [39,40]. These cells were also sensitive to inhibition with the MEK inhibitor, AZD6244 in the presence or absence of vemurafenib, suggesting that in this cell clone oncogenic N-RAS confers resistance by principally engaging the MAPK cascade. Oncogenic N-RAS mutations were also detected in a patient with acquired resistance to vemurafenib; separate N-RAS mutations (Q61K and Q61R) were detected in two melanoma biopsies taken on initial progression and six months after initial progression. Both mutations were associated with copy-number amplification and N-RAS overexpression [29]. 
In a second study, oncogenic N-RAS ${ }^{\mathrm{Q} 61 \mathrm{H}}$ was detected in two of six dabrafenib resistant subclones, generated from the MelRMu cell line. In contrast to the initial report [29], these two N-RAS mutant, MelRMu sublines showed diminished sensitivity to MEK inhibitor, trametinib and to the combined inhibition of BRAF and MEK, when compared to the parental cells. Moreover, ectopic expression of $\mathrm{N}-\mathrm{RAS}^{\mathrm{Q} 61 \mathrm{~K}}$ in the MelRMu cells diminished the efficacy of combined MEK and BRAF inhibition [38]. A third report also identified N-RAS mutations (N-RASQ61K and N-RAS ${ }^{\mathrm{A} 146 \mathrm{~T}}$ ) in two melanoma sublines with acquired resistance to dabrafenib. These mutations were shown to confer dabrafenib resistance, and induced the heterodimerization of BRAFV600E with C-RAF in the presence of drug [41]. These N-RAS mutant clones showed partial sensitivity to trametinib and to the concurrent inhibition of BRAF and MEK proteins [41]. It is known that mutant N-RAS can signal via multiple pathways including the PI3K/AKT/mTOR survival cascade [42] and consequently, N-RAS mutant dabrafenib-resistant melanoma cells were responsive to the simultaneous inhibition of MEK and the $\mathrm{PI} 3 \mathrm{~K} / \mathrm{mTOR}$ pathway [41].

There are some discrepancies in the literature regarding the role of activated RAS in selectively sensitizing cancer cells to MEK inhibition. Certainly, N-RAS mutation status did not predict MEK inhibitor sensitivity in melanoma cell lines [43], and MEK inhibitors show only modest clinical activity in patients with RAS-mutant tumors [9, 44]. It seems likely that the impact of mutant N-RAS on MEK inhibitor responses reflects its expression and activity and ultimately the network of activated N-RAS-dependent effectors. This is in agreement with a recent report demonstrating that $\mathrm{K}-\mathrm{RAS}^{13 \mathrm{D}}$-mutant HCT116 colorectal cancer cells became resistant to MEK inhibition upon amplification of the driving K-RAS ${ }^{13 \mathrm{D}}$ oncogene [45].

\section{CRAF overexpression}

Increased expression of the CRAF kinase has also been associated with BRAF inhibitor resistance (Figure 2). Villanueva et al. (2010) observed increased CRAF protein levels in melanoma cells chronically treated with the BRAF inhibitor SB-590885. In this cell model MAPK signalling driven by persistent insulin growth factor receptor (IGF-1R) activity, was rewired to utilise both CRAF and ARAF (Figure 1), and the inhibition of all three RAF isoforms was required to inhibit the proliferation of these 885-resistant cells [46]. This is in contrast to melanoma sublines rendered resistant to the pan-RAF inhibitor AZ628. These AZ628-resistant cells showed elevated basal levels of CRAF protein, but the knockdown of CRAF alone strongly inhibited cell proliferation, in the absence of AZ628 treatment [47]. These cells switched from BRAF to CRAF dependence, and the precise mechanism of CRAF-mediated AZ628 resistance remains unclear, as this inhibitor strongly suppresses both BRAFV600E and CRAF [48]. The role of CRAF in conferring RAF-inhibitor resistance may reflect the distinct genetic profiles of the melanoma cells used, the pathway rewiring involved in resistance, the mechanism of drug action and its impact on the RAF protein dimerization. 


\section{MEK mutations}

Mutations in mitogen activated protein kinase, MEK1 have also been shown to confer resistance to MAPK inhibitors. A random mutagenesis screen of MEK1 revealed that mutations interfering with target-drug binding (e.g. I99T, G128D, L215P) and mutations that upregulate MEK1 intrinsic activity (e.g. Q56P, P124S) conferred resistance to the allosteric MEK inhibitor AZD6244 [49]. The G128D MEK1 mutation also conferred resistance to the BRAF inhibitor PLX4720 [50] (Figure 5).
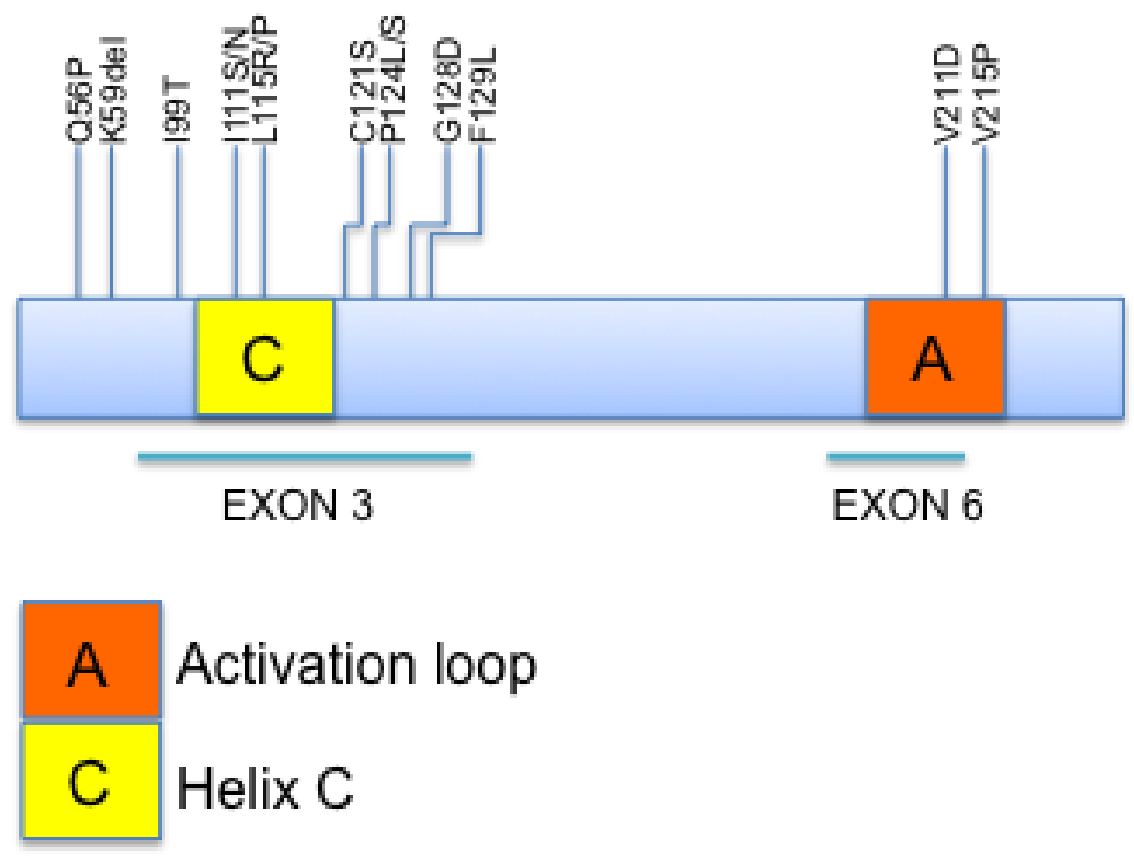

Figure 5. MEK1 mutations associated with MAPK inhibitor resistance. Allosteric MEK inhibitors binds to the MEK1 hydrophobic pocket that includes residues from helix $C$ and the activation loop. Primary MEK1 mutations affect this drug-binding pocket (e.g. 199T, I111N/S, L115P/R, G128D, F129L, V211D and L215P) and can directly perturb the allosteric binding of the MEK inhibitor. Secondary MEK1 mutations reside outside the drug-binding region and include mutations near the amino terminus (e.g. Q56P) and proximal to the helix C (C121S, P124S/L). These secondary MEK1 mutations increase MEK1 kinase activity. The C121S and P124L MEK1 mutation have been detected in MEK inhibitor resistant patient tumors [50], whereas P124S and I111S MEK1 mutations were identified in pre-treatment melanomas [54]. Shi et al. found that of the P124S, I111S and C121S, only C121S conferred vemurafenib resistance in melanoma cells.

Deep sequencing of tumors from five patients progressing on AZD6244 treatment, identified the MEK $1^{\mathrm{P} 124 \mathrm{~L}}$ mutation in the progressing, but not pre-treatment tumor sample from one patient. The existence of this MEK1 mutation was independently verified in ex vivo cell lines established from tumor material, and its activity in conferring MEK- and BRAF-inhibitor re- 
sistance validated in transfected melanoma cells. As with BRAF truncation and amplification, alterations in MEK1 protein did not alter the sensitivity of melanoma cells to the combined inhibition of BRAF and MEK inhibitors. A MEK $1^{\mathrm{C} 121 \mathrm{~S}}$ mutation was detected via mutational profiling in a melanoma sample from a patient with acquired resistance to vemurafenib. This mutation was not detected in the pre-treatment biopsy, showed increased intrinsic kinase activity and conferred resistance to BRAF and MEK inhibition in vitro [50]. BRAFi-resistant YUSIT1 melanoma cells also acquired a MEK1 mutation with increased kinase activity (K59del). These cells were dependent on MEK1 for proliferation and displayed higher ERK phosphorylation following treatment with dabrafenib [41].

Resistance to the allosteric MEK inhibitor PD0325901 in breast and colorectal cancer cell lines was also consistently associated with MEK mutations in the allosteric binding domain. MEK-inhibitor resistant sublines derived from the MDA-MB-231 breast and HCT-116 colon cancer cells gained the MEK1 $1^{\mathrm{L} 115 \mathrm{P}}$ and MEK1 ${ }^{\mathrm{F} 129 \mathrm{~L}}$ mutations, respectively and the MEK-inhibitor resistant LoVo colorectal cells acquired a MEK2 ${ }^{\mathrm{V} 215 \mathrm{E}}$ mutation (homologous to V211D mutations in MEK1) (Figure 5). The L115P and V211D mutations abrogated MEK inhibitor binding, while F129L increased the intrinsic activity of MEK and showed enhanced interaction with CRAF $[51,52]$. Cell lines expressing mutant MEK1 ${ }^{\mathrm{K} 57 \mathrm{~N}}$, which was identified in two lung adenocarcinomas, also showed decreased sensitivity to MEK inhibition [53].

A recent study found that MEK1 mutations identified in resistant melanoma lesions might not predict BRAF-inhibitor sensitivity. Shi et al found that five of 31 melanomas excised pre-BRAF inhibitor treatment carried concurrent somatic BRAF and MEK1 $\left(\mathrm{MEK} 1^{\mathrm{P} 124 \mathrm{~S}}\right.$ and $\mathrm{MEK}^{\mathrm{I111S}}$ ) activating mutations and that three of these five patients showed objective tumor responses. When the P124S, I111S and C121S MEK1 mutants were stably introduced into a series of melanoma cell lines, only the MEK1 ${ }^{\mathrm{C} 121 \mathrm{~S}}$ mutant restored p-ERK levels in the presence of vemurafenib, even though all mutants showed intrinsically enhanced kinase activity [54] (Figure 5). Thus, the relative impact of MEK1 mutations may vary depending on the type of mutation, tumor genetic background and the dependence on BRAF. For instance, YUSIT1 cells were dependent on the MEK1 ${ }^{\text {K59del }}$ for proliferation, and the MEK1 ${ }^{\mathrm{F} 129 \mathrm{~L}}$ mutant may induce a BRAF to CRAF kinase switch $[41,51]$. Finally, a more detailed tumor profile, correlating tumor response with the relative proportion of double-BRAF/MEK1 mutant cells within metastases, will help clarify the precise role of MEK1 in mediating BRAF-inhibitor resistance.

\section{COT overexpression}

A recent gain of function screen tested the activity of 597 kinases ( $75 \%$ of the annotated human kinases) in conferring vemurafenib resistance in the A375 melanoma cell line. Nine candidates, including receptor tyrosine kinases (Axl, ERBB2), conferred significant resistance with the mitogen activated protein kinase kinase kinase 8 (the gene encoding COT/Tpl2) emerging as the top candidate. Overexpression of COT resulted in constitutive ERK activation in the presence of vemurafenib (Figure 2). COT activated ERK via MEK-dependent and 
-independent mechanisms and ectopic COT expression conferred decreased sensitivity to the MEK inhibitors CI-1040 and AZD6244. COT expression was also elevated in two of three patient samples obtained early in the course of treatment and further increased in a relapsing specimen relative to its pre-treatment and on-treatment controls. Considering that inhibition of BRAF ${ }^{\mathrm{V} 600 \mathrm{E}}$ increases COT expression, it is possible that COT accumulation may reflect secondary responses to BRAF inhibition and resistance. Nevertheless, the silencing and inhibition of COT in the RPMI-7951 melanoma cells, which express increased COT, decreased ERK phosphorylation and suppressed cell viability [55].

\section{Activation of receptor tyrosine kinases}

An emerging theme in BRAF inhibitor resistance is the upregulation and activation of receptor tyrosine kinases. Garraway and co-workers demonstrated that ectopically expressed receptor tyrosine kinases Axl and ERBB2, circumvented vemurafenib activity [55], and two independent reports detected increased expression and activity of the platelet derived growth factor (PDGFRß) and IGF-1R in vemurafenib-resistant melanoma sublines [29, 46] (Figure 2). Vemurafenib-resistant tumor biopsies derived from patients also showed overexpression of PDGFRß (4 of 11 patients) and IGF-1R ( 2 of 5 patients) compared to the corresponding pre-treatment tumor specimens $[29,46]$. The knockdown or inhibition of PDGFRß and IGF-1R overcame vemurafenib resistance in cell lines, but resistance was not due to activation of ERK alone [29, 46]. Instead, receptor tyrosine kinase-upregulated, vemurafenib resistant melanoma cell lines, showed phosphorylation of both ERK and PI3K/AKT (Figure 2), and the concurrent and sustained inhibition of the MAPK and PI3K/AKT/mTOR pathways was required to overcome PDGFRß- and IGF-1R-mediated vemurafenib resistance [46, 56]. The upregulation and activation of these receptors was not due to gene amplification or genetic alterations within the coding sequence $[29,46]$.

These studies predict that RTK activation via increased autocrine tumor cell ligand secretion, or paracrine ligand production from stromal cells may confer resistance to MAPK inhibition. A recent report found increased activation of the fibroblast growth factor (FGF) receptor 3 was associated with elevated levels of autocrine secreted FGF2 ligand in vemurafenib resistant melanoma sublines [57]. Moreover the secretion of growth factors from cocultured fibroblast cells conferred vemurafenib resistance to BRAF-mutant melanoma cell lines. Stromal cell secretion of the hepatocyte growth factor (HGF) correlated best with vemurafenib resistance in this cell screen, and HGF was detected in tumor-associated stromal cells in 23 of 34 melanoma tumors resected from patients prior to MAPK inhibitor treatment. Critically, these 23 patients also showed activation of the HGF receptor MET in their tumor biopsies, and had a poorer response to MAPK-inhibitor treatment compared to patients whose stromal cells lacked HGF expression [58]. Increased plasma HGF levels in 126 metastatic melanoma patients, prior to treatment with vemurafenib, was also associated with a reduction in the progression-free and overall survival rates [59]. The stromal cell secretion of HGF resulted in reactivation of the MAPK and PI3K/mTOR signalling pathways and immediate (innate) resistance to RAF inhibition. Whether activation of MET also has a role in ac- 
quired resistance to RAF inhibitors remains to be determined, but activating somatic MET mutations and amplifications have been detected in human cancers [60-62]. Regardless, of the mechanism of MET activation, the sensitivity of MET-activated melanoma cells can be restored by the simultaneous inhibition of RAF and either HGF or MET [58]. Finally, it is worth noting that although activation of PDGFRß and IGF-1R are associated with vemurafenib resistance $[29,46]$, the ligand activation of these two receptors appears insufficient to drive sustained pathway activation or vemurafenib resistance $[58,59]$.

\section{Other regulators of response to MAPK inhibitors}

Typically, the suppression of MAPK signalling promotes cell cycle arrest that is associated with increased expression of the CDK inhibitor p27 $^{\text {Kip1 }}$ and inhibition of cyclin D1 expression (Figure 1). Cyclin D1 is a regulator of the cyclin dependent kinases (CDKs) 4 and 6 and the formation of binary cyclin D-CDK4/6 complexes promote the phosphorylation of the retinoblastoma protein $(\mathrm{pRb})$ and cell cycle progression [63-65]. Cyclin D1 is commonly amplified in melanoma and often in conjunction with mutated BRAF [66, 67]. The clinical significance of this genotype was demonstrated in BRAF-mutant melanoma cell lines with increased cyclin D1 protein expression. These cells showed intrinsic resistance to the growth-arresting effects of the RAF inhibitor, SB590885 and the ectopic expression of cyclin D1 conferred RAF-inhibitor resistance, which was enhanced by the dual overexpression of CDK4 and cyclin D1 [67]. These data confirm that the MAPK-independent expression of critical MAPK downstream targets will regulate RAF-inhibitor response and may diminish the dependence of cells to oncogenic BRAF.

Several independent studies have shown that loss of the phosphatase and tensin homo$\log$ (PTEN) tumor suppressor, which occurs in over $10 \%$ of melanoma tumors, is predictive of attenuated RAF-inhibitor mediated cytotoxicity [68, 69]. Cells lacking PTEN remain dependent on MAPK for proliferation but utilise increased AKT signalling for survival (Figure 2). Elevated AKT promotes the nuclear exclusion of the FOXO3a transcription factor, which leads to the downstream suppression of the FOXO3a pro-apoptotic target BIM [69, 70]. Predictably, ectopic expression of activated AKT3 also prevented BRAF inhibitor induced BIM and apoptosis [71] and MEK inhibitor-sensitive cancer cell lines show significantly higher FOXO3a and BIM protein levels compared to resistant cell lines [70]. Similar to RTK-induced resistance, the simultaneous inhibition of the MAPK and AKT pathways is required to restore PTEN-null cell sensitivity to MAPK inhibitors [68]. Finally, homozygous PTEN loss and increased pAKT levels were associated with vemurafenib resistance in a progressing biopsy derived from a single patient [46].

Considering the independent roles of cyclin D and PTEN in diminishing dependence on MAPK signalling and engaging the AKT survival cascade, it is anticipated that the concurrent alteration of these cell cycle regulators would confer increased levels of resistance to MAPK inhibitors. In a recent study, vemurafenib was shown to have purely cytostatic effects in melanoma cells with either PTEN or pRb loss; pRb deleted cells 
should behave as cells with elevated cyclinD1/CDK4 overexpression. BRAF ${ }^{\mathrm{V} 600 \mathrm{E}}$ cells with concurrent loss of both $\mathrm{pRb}$ and PTEN were completely resistant to RAF inhibition, and these cells continued proliferating in the presence of this RAF inhibitor [68]. The clinical significance of $\mathrm{pRb}$ loss in conferring MAPK inhibitor resistance is uncertain, however, as $\mathrm{pRb}$ loss is uncommon in melanoma [72].

Finally, activation of the STAT3 pathway was found to be associated with AZD6244 resistance in a panel of lung cancer cell lines. STAT3 activity was shown to decrease BIM accumulation through the upregulation of miR-17, and the inhibition of STAT3 or miR-17 upregulated BIM and sensitized resistant cells to MEK inhibition [73].

\section{Therapies to overcome MAPK inhibitor resistance}

Irrespective of the precise mechanisms of resistance to class I RAF inhibitors, tumors that acquire resistance or are inherently insensitive to these inhibitors often maintain some dependency on the MAPK pathway [29, 33, 38, 55, 57, 74]. These data suggest that further inhibition of the MAPK cascade at the downstream MEK or ERK nodes may be effective in treating resistance to single agent BRAF inhibitors. Despite the preclinical evidence of MEK-inhibitor sensitivity in cells with acquired resistance to BRAF inhibitors [38], clinical trials applying this strategy have been disappointing. The MEK inhibitor trametinib showed minimal activity (response rates of $3 \%$ ) in patients previously treated with a BRAF inhibitor [75]. Clinical benefit was observed, however, when patients who progressed on prior BRAF inhibitor were treated with a combination of BRAF and MEK inhibitors. Partial responses were observed in $17 \%$ of patients, suggesting that dual MAPK blockade can abrogate some BRAF inhibitor resistance mechanisms [76]. The triaging of patients, based on BRAF inhibitor resistance drivers, may also improve the clinical benefit of second line MAPK inhibitor therapies. For instance, melanoma cells expressing BRAF splice variants are sensitive to MEK inhibition [33], whereas cells with BRAF copy number gains respond to the concurrent inhibition of BRAF and MEK [30, 31]. Finally, specific inhibitors of ERK have recently become available, and these show anti-proliferative activity in MEK-inhibitor resistant cells and synergise with MEK inhibitors to prevent or delay the emergence of acquired resistance [52].

Sustained and significant responses have also been observed when RAF-inhibitor resistant cell lines are treated with combination MAPK and PI3K/mTOR inhibitors. For instance, in RTK-expressing vemurafenib-resistant cells, inhibition of PI3K/mTOR activity in combination with vemurafenib showed potent synergy. Compensatory signalling via MEK permitted survival in the presence of PI3K/mTOR/MAPK inhibition, but cytotoxicity was restored using a combination of MEK inhibitor with the dual PI3K/mTOR inhibitor BEZ235 [56]. A number of combinations of MEK and PI3K/mTOR pathway inhibitors combinations have entered early phase clinical trials, however their benefit in the setting of BRAF/MEK inhibitor resistance remains untested. 
Many of the proteins involved in melanoma development and RAF-inhibitor resistance are targets of the heat shock protein (Hsp)-90 family of chaperones. Hsp90 proteins regulate the conformation, stability and function of many RTKs and kinases, including IGF-1R, BRAF, CRAF, CDK4, AKT and cyclin D1 [77, 78]. The pharmacological inhibition of Hsp90 using the selective inhibitor, XL888 abrogated acquired and intrinsic vemurafenib resistance. XL888 induced apoptosis in melanoma cells with mutant N-RAS, elevated PDGFRß, COT, IGF-1R, CRAF and cyclin D1. Apoptosis was associated with diminished accumulation of the resistance driver, nuclear accumulation of FOXO3a and an increase in BIM expression. Moreover, Hsp90 inhibition was a more effective apoptotic inducer when combined with MEK and PI3K inhibition [79]. Hsp90 inhibitors have shown promising results in ERBB2-amplified breast cancers [80], but lacked clinical activity in vemurafenib-naive melanoma patients [81]. Evaluation of pre- and post treatment melanoma biopsies confirmed incomplete degradation of $\mathrm{BRAF}^{\mathrm{V} 600 \mathrm{E}}$, when the inhibitor was given on a weekly schedule. Whether Hsp90 inhibition will prove effective when administered more frequently, in RAF-inhibitor resistant melanoma patients, or in combination with MAPK inhibitors remains to be tested.

\section{Conclusions}

BRAF-targeted therapy has recently emerged as the standard treatment for patients with BRAF-mutant melanoma. Responses are not durable, however and studies of acquired resistance to BRAF inhibition reveal a diversity of resistance mechanism but a common resistance theme. Melanoma cells adapt by re-engaging MAPK signalling and activating parallel survival networks. The management and prevention of BRAF inhibitor resistance is likely to be achieved through combination therapies. The combination of BRAF and MEK inhibitors has shown better response than single agent therapy [25] and is currently being evaluated in phase III clinical trials compared to vemurafenib (NCT01597908) or dabrafenib (NCT01584648) in treatment naive patients with BRAFV600E mutant melanoma. Trials combining MEK with AKT inhibitors (NCT01021748), the pan-RAF inhibitor sorafenib and MEK inhibition (NCT0034999206), testing HDAC inhibition with vorinostat (NCT006670820) are also under way. Further, Phase I trials for inhibition of PDGFR $\beta$, FGFR and other tyrosine kinases using Dovitinib in patients with advanced melanoma has shown promising results [82]. Finally, rechallenging patients with selective BRAF inhibitors after a treatment-free interval provided clinical benefit to two patients who had previously progressed on MAPK inhibitors [83]. Additional studies are required to determine the significance of rechallenging patients after treatment interruption.

It has been suggested that a detailed catalogue of resistance mechanism in an individual's tumor should inform effective second line therapy [84]. This strategy may not prove sufficient, as it does not account for stromal-mediated resistance drivers, the heterogeneous nature of melanoma and the fact that melanoma tumors from a single patient may develop multiple mechanisms of resistance. For instance, two independent vemurafenib-resistant nodal metastases derived from a single patient, harboured distinct N-RAS activating mutations 
[29] and intra-tumoral heterogeneity has been observed in a progressing BRAF-mutant melanoma metastases from patients treated with BRAF inhibitors $[85,86]$.

Nevertheless, defining the mechanisms of RAF-inhibitor resistance is a critical step in understanding the signalling pathways required to circumvent therapy. At present, up to $40 \%$ of RAF-inhibitor resistant melanomas have undefined resistance drivers, and the role of MAPK and PI3K signalling needs to be assessed in this subgroup. The fact that half of all melanoma patients have wild type BRAF melanoma, further highlights the need for an integrated preclinical and clinical approach to guide rational design of effective initial and second-line treatment options.

\section{Acknowledgements}

This work is supported by Program Grant 633004 and project grants of the National Health and Medical Research Council of Australia (NHMRC) and an infrastructure grant to Westmead Millennium Institute by the Health Department of NSW through Sydney West Area Health Service. HR is a recipient of a Cancer Institute New South Wales, Research Fellowship and a NHMRC Senior Research Fellowship. MSC is supported by a Rotary Health Australia scholarship

\section{Author details}

Kavitha Gowrishankar, Matteo S. Carlino and Helen Rizos

*Address all correspondence to: helen.rizos@sydney.edu.au

Westmead Institute for Cancer Research, University of Sydney at Westmead Millennium Institute and Melanoma Institute Australia, Westmead Hospital, Westmead, Australia

\section{References}

[1] Jemal A, Siegel R, Xu J, Ward E. Cancer statistics, 2010. CA Cancer J Clin. 2010;60(5): 277-300.

[2] Cummins DL, Cummins JM, Pantle H, Silverman MA, Leonard AL, Chanmugam A. Cutaneous malignant melanoma. Mayo Clin Proc. 2006;81(4):500-7.

[3] Balch CM, Gershenwald JE, Soong SJ, Thompson JF, Atkins MB, Byrd DR, et al. Final version of 2009 AJCC melanoma staging and classification. J Clin Oncol. 2009;27(36): 6199-206.. 
[4] Thompson JF, Scolyer RA, Kefford RF. Cutaneous melanoma. Lancet. 2005;365:687-701.

[5] Davies H, Bignell GR, Cox C, Stephens P, Edkins S, Clegg S, et al. Mutations of the BRAF gene in human cancer. Nature. 2002;417(6892):949-54.

[6] Lee JH, Choi JW, Kim YS. Frequencies of BRAF and NRAS mutations are different in histological types and sites of origin of cutaneous melanoma: a meta-analysis. Br J Dermatol. 2011;164(4):776-84.

[7] Wan PT, Garnett MJ, Roe SM, Lee S, Niculescu-Duvaz D, Good VM, et al. Mechanism of activation of the RAF-ERK signaling pathway by oncogenic mutations of BRAF. Cell. 2004;116(6):855-67.

[8] Flaherty KT, Puzanov I, Kim KB, Ribas A, McArthur GA, Sosman JA, et al. Inhibition of mutated, activated BRAF in metastatic melanoma. N Engl J Med. 2010;363(9): 809-19.

[9] Falchook GS, Lewis KD, Infante JR, Gordon MS, Vogelzang NJ, Demarini DJ, et al. Activity of the oral MEK inhibitor trametinib in patients with advanced melanoma: a phase 1 dose-escalation trial. Lancet Oncol. 2012;13(8):782-9.

[10] Hauschild A, Grob JJ, Demidov LV, Jouary T, Gutzmer R, Millward M, et al. Dabrafenib in BRAF-mutated metastatic melanoma: a multicentre, open-label, phase 3 randomised controlled trial. Lancet. 2012;380(9839):358-65.

[11] Chapman PB, Hauschild A, Robert C, Haanen JB, Ascierto P, Larkin J, et al. Improved Survival with Vemurafenib in Melanoma with BRAF V600E Mutation. N Engl J Med. 2011;364:2507-16.

[12] Sosman JA, Kim KB, Schuchter L, Gonzalez R, Pavlick AC, Weber JS, et al. Survival in BRAF V600-mutant advanced melanoma treated with vemurafenib. N Engl J Med. 2012;366(8):707-14.

[13] Long GV, Trefzer U, Davies MA, Kefford RF, Ascierto PA, Chapman PB, et al. Dabrafenib in patients with Val600Glu or Val600Lys BRAF-mutant melanoma metastatic to the brain (BREAK-MB): a multicentre, open-label, phase 2 trial. Lancet Oncol. 2012 (in press).

[14] Dummer R, Rinderknecht J, Goldinger S, Wagner I, Mitchell L, Veronese M, et al. An open-label pilot study of vemurafenib in previously treated metastatic melanoma patients with brain metastases. J Clin Oncol. 2011;29:(suppl; abstr 8548).

[15] Falchook GS, Long GV, Kurzrock R, Kim KB, Arkenau TH, Brown MP, et al. Dabrafenib in patients with melanoma, untreated brain metastases, and other solid tumours: a phase 1 dose-escalation trial. Lancet. 2012;379:1893-901.

[16] Platz A, Egyhazi S, Ringborg U, Hansson J. Human cutaneous melanoma; a review of NRAS and BRAF mutation frequencies in relation to histogenetic subclass and body site. Mol Oncol. 2008;1(4):395-405. 
[17] Curtin JA, Busam K, Pinkel D, Bastian BC. Somatic activation of KIT in distinct subtypes of melanoma. Journal of clinical oncology : official journal of the American Society of Clinical Oncology. 2006;24(26):4340-6.

[18] Krauthammer M, Kong Y, Ha BH, Evans P, Bacchiocchi A, McCusker JP, et al. Exome sequencing identifies recurrent somatic RAC1 mutations in melanoma. Nat Genet. 2012;29(10).

[19] Pollock PM, Harper UL, Hansen KS, Yudt LM, Stark M, Robbins CM, et al. High frequency of BRAF mutations in nevi. Nat Genet. 2003;33(1):19-20.

[20] Hingorani SR, Jacobetz MA, Robertson GP, Herlyn M, Tuveson DA. Suppression of BRAF(V599E) in human melanoma abrogates transformation. Cancer Res. 2003;63:5198-202.

[21] Karasarides M, Chiloeches A, Hayward R, Niculescu-Duvaz D, Scanlon I, Friedlos F, et al. B-RAF is a therapeutic target in melanoma. Oncogene. 2004;23(37):6292-8.

[22] Hoeflich KP, Gray DC, Eby MT, Tien JY, Wong L, Bower J, et al. Oncogenic BRAF is required for tumor growth and maintenance in melanoma models. Cancer Res. 2006;66(2):999-1006.

[23] Sumimoto H, Miyagishi M, Miyoshi H, Yamagata S, Shimizu A, Taira K, et al. Inhibition of growth and invasive ability of melanoma by inactivation of mutated BRAF with lentivirus-mediated RNA interference. Oncogene. 2004;23(36):6031-9.

[24] Flaherty KT, Robert C, Hersey P, Nathan P, Garbe C, Milhem M, et al. Improved survival with MEK inhibition in BRAF-mutated melanoma. N Engl J Med. 2012;367(2): 107-14.

[25] Flaherty KT, Infante JR, Daud A, Gonzalez R, Kefford RF, Sosman J, et al. Combined BRAF and MEK Inhibition in Melanoma with BRAF V600 Mutations. N Engl J Med. 2012 (in press)

[26] Kefford R, Arkenau H-T, Brown MP, Millward M, Infante J, Long G, et al., editors. Selective inhibition of oncogenic BRAF V600E/K/D by GSK2118436: Evidence of clinical activity in subjects with metastatic melanoma. 7th International Melanoma Research Congress; 2010; Sydney, Australia.

[27] Ellis LM, Hicklin DJ. Resistance to Targeted Therapies: Refining Anticancer Therapy in the Era of Molecular Oncology. Clin Cancer Res. 2009;15(24):7471-8.

[28] Bixby D, Talpaz M. Seeking the causes and solutions to imatinib-resistance in chronic myeloid leukemia. Leukemia. 2011;25(1):7-22.

[29] Nazarian R, Shi H, Wang Q, Kong X, Koya RC, Lee H, et al. Melanomas acquire resistance to B-RAF(V600E) inhibition by RTK or N-RAS upregulation. Nature. 2010;468(7326):973-7. 
[30] Shi H, Moriceau G, Kong X, Lee MK, Lee H, Koya RC, et al. Melanoma whole-exome sequencing identifies (V600E)B-RAF amplification-mediated acquired B-RAF inhibitor resistance. Nat Commun. 2012;3(724):724.

[31] Corcoran RB, Dias-Santagata D, Bergethon K, Iafrate AJ, Settleman J, Engelman JA. BRAF Gene Amplification Can Promote Acquired Resistance to MEK Inhibitors in Cancer Cells Harboring the BRAF V600E Mutation. Sci Signal. 2010;3(149):ra84.

[32] Diaz LA, Jr., Williams RT, Wu J, Kinde I, Hecht JR, Berlin J, et al. The molecular evolution of acquired resistance to targeted EGFR blockade in colorectal cancers. Nature. 2012;486(7404):537-40.

[33] Poulikakos PI, Persaud Y, Janakiraman M, Kong X, Ng C, Moriceau G, et al. RAF inhibitor resistance is mediated by dimerization of aberrantly spliced BRAF(V600E). Nature. 2011;480:387-90.

[34] Weber CK, Slupsky JR, Kalmes HA, Rapp UR. Active Ras induces heterodimerization of cRaf and BRaf. Cancer Res. 2001;61(9):3595-8.

[35] Heidorn SJ, Milagre C, Whittaker S, Nourry A, Niculescu-Duvas I, Dhomen N, et al. Kinase-dead BRAF and oncogenic RAS cooperate to drive tumor progression through CRAF. Cell. 2010;140(2):209-21.

[36] Poulikakos PI, Zhang C, Bollag G, Shokat KM, Rosen N. RAF inhibitors transactivate RAF dimers and ERK signalling in cells with wild-type BRAF. Nature. 2010;464(7287):427-30.

[37] Hatzivassiliou G, Song K, Yen I, Brandhuber BJ, Anderson DJ, Alvarado R, et al. RAF inhibitors prime wild-type RAF to activate the MAPK pathway and enhance growth. Nature. 2010;464(7287):431-5.

[38] Gowrishankar K, Snoyman S, Pupo GM, Becker TM, Kefford RF, Rizos H. Acquired Resistance to BRAF Inhibition Can Confer Cross-Resistance to Combined BRAF/MEK Inhibition. J Invest Dermatol. 2012;132(7):1850-9.

[39] Dumaz N, Hayward R, Martin J, Ogilvie L, Hedley D, Curtin JA, et al. In melanoma, RAS mutations are accompanied by switching signaling from BRAF to CRAF and disrupted cyclic AMP signaling. Cancer Res. 2006;66(19):9483-91.

[40] Jaiswal BS, Janakiraman V, Kljavin NM, Eastham-Anderson J, Cupp JE, Liang Y, et al. Combined targeting of BRAF and CRAF or BRAF and PI3K effector pathways is required for efficacy in NRAS mutant tumors. PLoS ONE. 2009;4(5):e5717.

[41] Greger J, Eastman S, Zhang V, Bleam MR, Hughes A, Smitheman KN, et al. Combinations of BRAF, MEK, and PI3K/mTOR inhibitors overcome acquired resistance to the BRAF inhibitor GSK2118436 dabrafenib, mediated by NRAS or MEK mutations. Mol Cancer Ther. 2012;11(4):909-20.

[42] Smalley KS. Understanding melanoma signaling networks as the basis for molecular targeted therapy. J Invest Dermatol. 2010;130(1):28-37. 
[43] Solit DB, Garraway LA, Pratilas CA, Sawai A, Getz G, Basso A, et al. BRAF mutation predicts sensitivity to MEK inhibition. Nature. 2006;439(7074):358-62.

[44] Nissan MH, Solit DB. The "SWOT" of BRAF Inhibition in Melanoma: RAF Inhibitors, MEK Inhibitors or Both? Curr Oncol Rep. 2011;13(6):479-87.

[45] Little AS, Balmanno K, Sale MJ, Newman S, Dry JR, Hampson M, et al. Amplification of the Driving Oncogene, KRAS or BRAF, Underpins Acquired Resistance to MEK1/2 Inhibitors in Colorectal Cancer Cells. Sci Signal. 2011;4(166):ra17.

[46] Villanueva J, Vultur A, Lee JT, Somasundaram R, Fukunaga-Kalabis M, Cipolla AK, et al. Acquired resistance to BRAF inhibitors mediated by a RAF kinase switch in melanoma can be overcome by cotargeting MEK and IGF-1R/PI3K. Cancer Cell. 2010;18(6):683-95.

[47] Montagut C, Sharma SV, Shioda T, McDermott U, Ulman M, Ulkus LE, et al. Elevated CRAF as a potential mechanism of acquired resistance to BRAF inhibition in melanoma. Cancer Res. 2008;68(12):4853-61.

[48] Shen M, Wu A, Aquila B, Lyne P, Drew L, editors. Linking molecular characteristics to the pharmacological response of a panel of cancer cell lines to the BRAF inhibitor, AZ628. AACR 98th Meeting; 2007 June 9, 2011; Los Angeles.

[49] Emery CM, Vijayendran KG, Zipser MC, Sawyer AM, Niu L, Kim JJ, et al. MEK1 mutations confer resistance to MEK and B-RAF inhibition. Proc Natl Acad Sci U S A. 2009;106(48):20411-6.

[50] Wagle N, Emery C, Berger MF, Davis MJ, Sawyer A, Pochanard P, et al. Dissecting Therapeutic Resistance to RAF Inhibition in Melanoma by Tumor Genomic Profiling. J Clin Oncol. 2011;29(22):3085-96.

[51] Wang H, Daouti S, Li WH, Wen Y, Rizzo C, Higgins B, et al. Identification of the MEK1(F129L) activating mutation as a potential mechanism of acquired resistance to MEK inhibition in human cancers carrying the B-RafV600E mutation. Cancer Res. 2011;71(16):5535-45.

[52] Hatzivassiliou G, Liu B, O'Brien C, Spoerke JM, Hoeflich KP, Haverty PM, et al. ERK inhibition overcomes acquired resistance to MEK inhibitors. Mol Cancer Ther. 2012;11(5):1143-54.

[53] Marks JL, Gong Y, Chitale D, Golas B, McLellan MD, Kasai Y, et al. Novel MEK1 mutation identified by mutational analysis of epidermal growth factor receptor signaling pathway genes in lung adenocarcinoma. Cancer Res. 2008;68(14):5524-8.

[54] Shi H, Moriceau G, Kong X, Koya RC, Nazarian R, Pupo GM, et al. Preexisting MEK1 Exon 3 Mutations in V600E/KBRAF Melanomas Do Not Confer Resistance to BRAF Inhibitors. Cancer Discov. 2012;2(5):414-24. 
[55] Johannessen CM, Boehm JS, Kim SY, Thomas SR, Wardwell L, Johnson LA, et al. COT drives resistance to RAF inhibition through MAP kinase pathway reactivation. Nature. 2010;468:968-72.

[56] Shi H, Kong X, Ribas A, Lo RS. Combinatorial treatments that overcome PDGFRbetadriven resistance of melanoma cells to V600EB-RAF inhibition. Cancer Res. 2011;71(15):5067-74.

[57] Yadav V, Zhang X, Liu J, Estrem S, Li S, Gong XQ, et al. Reactivation of Mitogen-Activated Protein Kinase (MAPK) Pathway by FGF Receptor 3 (FGFR3)/Ras Mediates Resistance to Vemurafenib in Human B-RAF V600E Mutant Melanoma. J Biol Chem. 2012;287(33):28087-98.

[58] Straussman R, Morikawa T, Shee K, Barzily-Rokni M, Qian ZR, Du J, et al. Tumour micro-environment elicits innate resistance to RAF inhibitors through HGF secretion. Nature. 2012;487(7408):500-4.

[59] Wilson TR, Fridlyand J, Yan Y, Penuel E, Burton L, Chan E, et al. Widespread potential for growth-factor-driven resistance to anticancer kinase inhibitors. Nature. 2012 Jul 4;487(7408):505-9.

[60] Puri N, Ahmed S, Janamanchi V, Tretiakova M, Zumba O, Krausz T, et al. c-Met is a potentially new therapeutic target for treatment of human melanoma. Clin Cancer Res. 2007;13(7):2246-53.

[61] Lorenzato A, Olivero M, Patane S, Rosso E, Oliaro A, Comoglio PM, et al. Novel somatic mutations of the MET oncogene in human carcinoma metastases activating cell motility and invasion. Cancer Res. 2002;62(23):7025-30.

[62] Engelman JA, Zejnullahu K, Mitsudomi T, Song Y, Hyland C, Park JO, et al. MET amplification leads to gefitinib resistance in lung cancer by activating ERBB3 signaling. Science. 2007;316(5827):1039-43.

[63] Sherr CJ, Roberts JM. CDK inhibitors: positive and negative regulators of G1-phase progression. Genes Dev. 1999;13(12):1501-12.

[64] Lavoie JN, L'Allemain G, Brunet A, Muller R, Pouyssegur J. Cyclin D1 expression is regulated positively by the p42/p44MAPK and negatively by the p38/HOGMAPK pathway. J Biol Chem. 1996;271(34):20608-16.

[65] Donovan JC, Milic A, Slingerland JM. Constitutive MEK/MAPK activation leads to p27(Kip1) deregulation and antiestrogen resistance in human breast cancer cells. J Biol Chem. 2001;276(44):40888-95.

[66] Lazar V, Ecsedi S, Szollosi AG, Toth R, Vizkeleti L, Rakosy Z, et al. Characterization of candidate gene copy number alterations in the 11q13 region along with BRAF and NRAS mutations in human melanoma. Mod Pathol. 2009;22(10):1367-78. 
[67] Smalley KS, Lioni M, Dalla Palma M, Xiao M, Desai B, Egyhazi S, et al. Increased cyclin D1 expression can mediate BRAF inhibitor resistance in BRAF V600E-mutated melanomas. Mol Cancer Ther. 2008;7(9):2876-83.

[68] Xing F, Persaud Y, Pratilas CA, Taylor BS, Janakiraman M, She QB, et al. Concurrent loss of the PTEN and RB1 tumor suppressors attenuates RAF dependence in melanomas harboring (V600E)BRAF. Oncogene. 2012;31(4):446-57.

[69] Paraiso KH, Xiang Y, Rebecca VW, Abel EV, Chen A, Munko AC, et al. PTEN loss confers BRAF inhibitor resistance to melanoma cells through the suppression of BIM expression. Cancer Res. 2011;71:2750-60.

[70] Yang JY, Chang CJ, Xia W, Wang Y, Wong KK, Engelman JA, et al. Activation of FOXO3a is sufficient to reverse mitogen-activated protein/extracellular signal-regulated kinase kinase inhibitor chemoresistance in human cancer. Cancer Res. 2010;70(11):4709-18.

[71] Shao Y, Aplin AE. Akt3-mediated resistance to apoptosis in B-RAF-targeted melanoma cells. Cancer Res. 2010;70(16):6670-81.

[72] Saenz-Santamaria MC, McNutt NS, Shea CR. Immunohistochemical expression of retinoblastoma protein in cutaneous melanomas. Br J Dermatol. 1995;133(6):890-5.

[73] Dai B, Meng J, Peyton M, Girard L, Bornmann WG, Ji L, et al. STAT3 mediates resistance to MEK inhibitor through microRNA miR-17. Cancer Res. 2011;71(10):3658-68.

[74] Paraiso KH, Fedorenko IV, Cantini LP, Munko AC, Hall M, Sondak VK, et al. Recovery of phospho-ERK activity allows melanoma cells to escape from BRAF inhibitor therapy. Br J Cancer. 2010;102(12):1724-30.

[75] Kim KB, Lewis K, Pavlick AC, Infante JR, Ribas A, Sosman JA, et al., editors. A phase II study of the MEK1/MEK2 inhibitor GSK1120212 in metastatic BRAF-V600E or K mutant cutaneous melanoma patients previously treated with or without a BRAF inhibitor. International Melanoma Congress; 2011; Tampa, Florida, USA: Pigment Cell Res.

[76] Flaherty K, Infante JR, Falchook G, Weber JD, Daud A, Hamid O, et al., editors. Phase I/II expansion cohort of BRAF inhibitor GSK2118436 + MEK inhibitor GSK1120212 in patients with BRAF mutant metastatic melanoma who progressed on a prior BRAF inhibitor. International Melanoma Congress; 2011; Tampa, Florida, USA: Pigment Cell Res.

[77] Den RB, Lu B. Heat shock protein 90 inhibition: rationale and clinical potential. Ther Adv Med Oncol. 2012;4(4):211-8.

[78] Whitesell L, Santagata S, Lin NU. Inhibiting HSP90 to treat cancer: a strategy in evolution. Curr Mol Med. 2012;17:17. 
[79] Paraiso KH, Haarberg E, Wood E, Rebecca VW, Chen YA, Xiang Y, et al. The heat shock protein-90 inhibitor XL888 overcomes BRAF inhibitor resistance mediated through diverse mechanisms. Clin Cancer Res. 2012;18(9):2502-14.

[80] Modi S, Stopeck A, Linden H, Solit D, Chandarlapaty S, Rosen N, et al. HSP90 inhibition is effective in breast cancer: a phase II trial of tanespimycin (17-AAG) plus trastuzumab in patients with HER2-positive metastatic breast cancer progressing on trastuzumab. Clin Cancer Res. 2011;17(15):5132-9.

[81] Solit DB, Osman I, Polsky D, Panageas KS, Daud A, Goydos JS, et al. Phase II trial of 17-allylamino-17-demethoxygeldanamycin in patients with metastatic melanoma. Clin Cancer Res. 2008 Dec 15;14(24):8302-7.

[82] Nikolaou VA, Stratigos AJ, Flaherty KT, Tsao H. Melanoma: new insights and new therapies. J Invest Dermatol. 2012;132(3 Pt 2):854-63.

[83] Neyns B, Seghers AC, Wilgenhof S, Lebbe C. Successful rechallenge in two patients with BRAF-V600-mutant melanoma who experienced previous progression during treatment with a selective BRAF inhibitor. Melanoma Res. 2012 (in press).

[84] Alcala AM, Flaherty KT. BRAF Inhibitors for the Treatment of Metastatic Melanoma: Clinical Trials and Mechanisms of Resistance. Clin Cancer Res. 2012;18(1):33-9.

[85] Wilmott JS, Tembe V, Howle JR, Sharma R, Thompson JF, Rizos H, et al. Intratumoral molecular heterogeneity in a BRAF-mutant, BRAF inhibitor-resistant melanoma: a case illustrating the challenges for personalized medicine. Molecular cancer therapeutics. 2012 (in press)

[86] Richtig E, Schrama D, Ugurel S, Fried I, Niederkorn A, Massone C, et al. BRAF mutation analysis of only one single metastatic lesion can restrict the treatment of melanoma - a case report. Br J Dermatol. 2012;7(10):1365-2133.

[87] Catalanotti F, Solit DB. Will Hsp90 inhibitors prove effective in BRAF-mutant melanomas? Clin Cancer Res. 2012;18(9):2420-2. 